\title{
Resonance Gyrons and Quantum Geometry
}

\author{
Mikhail Karasev* \\ Moscow Institute of Electronics and Mathematics \\ karasev@miem.edu.ru
}

Dedicated to Hideki Omori

\begin{abstract}
We describe irreducible representations, coherent states and starproducts for algebras of integrals of motions (symmetries) of twodimensional resonance oscillators. We demonstrate how the quantum geometry (quantum Kähler form, metric, quantum Ricci form, quantum reproducing measure) arises in this problem. We specifically study the distinction between the isotropic resonance $1: 1$ and the general $l: m$ resonance for arbitrary coprime $l, m$. Quantum gyron is a dynamical system in the resonance algebra. We derive its Hamiltonian in irreducible representations and calculate the semiclassical asymptotics of the gyron spectrum via the quantum geometrical objects.
\end{abstract}

\section{Introduction}

For complicated dynamical systems, it is important to be able to abstract from studying concrete motions or states and to observe surrounding structures, like spaces, algebras, etc., which carry essential properties of the variety of motions in the whole.

\footnotetext{
*This work was partially supported by RFBR (grant 05-01-00918-a).
} 
For quantum (wave) systems, the standard accompanying mathematical structures are algebras of "observables," i.e., functions on phase spaces, and representations of these algebras in Hilbert vector spaces of "states." This is the starting viewpoint for the mathematical quantization theory [1]-10]. The more complicated systems are studied the more complicated algebras and phase spaces (symplectic manifolds) have to be used. Note that for general symplectic and even Kählerian manifolds the quantization problem is still unsolved.

It was demonstrated in [11, 12] that for general symplectic manifolds it is possible to approximate the symplectic potential by its quadratic part (the oscillator!), then to use this quadratic part in order to define the standard Groenewold-Moyal [13, 14 product on the tangent spaces, and to construct a formal *-product on the original manifold by a perturbation theory. Such oscillator-generated quantum manifolds were called the "Weyl manifolds" in [11.

In quantum and wave mechanics, one often meets a situation similar in certain sense: the dynamics of a system is, in general, chaotic, but there are some exclusive invariant submanifolds (for instance, equilibrium points) in the phase space around which the dynamics is regular and can be approximated by the oscillator motion in directions transversal to the submanifold. Thus the given system contains inside a built-in harmonic oscillator plus certain anharmonic part near the equilibrium:

$$
\frac{1}{2} \sum\left(p_{j}^{2}+\omega_{j}^{2} q_{j}^{2}\right)+\text { cubic }+ \text { quartic }+\ldots
$$

If the frequencies $\omega_{j}$ of the harmonic part are incommensurable (not in a resonance), then in a small neighborhood of the submanifold the anharmonic part just slightly perturbs these frequencies, and the whole motion is performed along the perturbed Liouville tori. This is the well-investigated situation both on the classical and quantum levels [15]-21].

If the frequencies $\omega_{j}$ are in a resonance then all standard approaches do not work and the picture occurs to be much more interesting from the viewpoint of quantum geometry. Here we will follow the works [22]-[25].

First of all, in the resonance case the Liouville tori are collapsed (to a smaller dimension), and the anharmonic part generates a nontrivial "averaged" motion in the new phase spaces: in the symplectic leave $\Omega$ of the commutant $\mathcal{F}_{\omega}$ of the harmonic part. The new phase spaces represent certain hidden dynamics committed to the resonance. This dynamics describes 
a precession of the parameters of the resonance harmonic motion under the action of the anharmonic part. We call this dynamical system a gyron (from the Greek word "gyro," i.e., "rotating").

In the simplest case of the isotropic 1:1 resonance for two degrees of freedom the gyron system is just the Euler top system from the theory of rigid body rotations, which is related to the linear Poisson brackets. For the general $l: m$ resonance, the gyron is described by a nonlinear Poisson brackets polynomial of degree $l+m-1$, see in 24, 25.

Of course, in the quantum case the resonance function algebra $\mathcal{F}_{\omega}$ has to be replaced by a resonance operator algebra $\mathfrak{F}_{\omega}$ which consists of operators commuting with the quantum oscillator $\frac{1}{2} \sum_{j}\left(\hat{p}_{j}^{2}+\omega_{j}^{2} q_{j}^{2}\right)$, where $\hat{p}_{j}=$ $-i \hbar \partial / \partial q_{j}$. This algebra is described by nonlinear commutation relations of polynomial type, see in [24, 25]. It is the dynamic algebra for quantum gyrons.

Note that there is a variety of important physical models containing inside the resonance Hamiltonians like (1.1). The quantum gyrons in these models can be considered as an analog of known quasiparticles similar to polarons, rotons, excitons, etc. ${ }^{1}$ As the simplest example, we mention the models of nano-physics (quantum dots, artificial atoms, quantum wires, see examples in [24]). Another example is the fiber waveguides in optics; they are described by the Hamiltonian

$$
p^{2}-n^{2}(q), \quad q, p \in \mathbb{R}^{3},
$$

where $n(q)$ is the refraction index having the maximum value along the waveguide axis, that is, along an arbitrary smooth curve in $\mathbb{R}^{3}$. The quadratic part of $n^{2}(q)$ in directions transversal to this curve is assumed to have commensurable frequencies in a certain resonance proportion $\omega_{1}: \omega_{2}=l: m$, where $l, m$ are coprime integers. The quantum gyron in this model describes certain hidden "polarization" of the light beam along the given curve in the optical medium, see in 24]. The propagation of such optical gyrons and their spectrum depend on the anharmonic part of the refraction index, and so one can control the properties of the gyron waves by changing the geometry of the curve just by bending the optical fiber.

The aim of the given paper is to describe the quantum geometry of the gyron phase spaces in the case of the $l: m$ resonance.

If $l=m=1$, then these phase spaces $\Omega$ are just homogeneous spheres $\mathbb{S}^{2}$, that is, the coadjoint $s u(2)$ orbits. The quantum geometry in this case

\footnotetext{
${ }^{1}$ Attention to this was paid by V. Maslov.
} 
coincides with the classical symplectic (Kählerian) geometry generated by linear Lie-Poisson brackets.

If at least one of the integers $l$ or $m$ exceeds 1 , then, as we will see below, the quantum geometry occurs to be unusual. The quantum phase spaces are still diffeomorphic to $\mathbb{S}^{2}$, but the classical symplectic form is singular on them. The correct symplectic (Kählerian) form and the reproducing measure of the quantum phase space are chosen from the nontrivial condition that the operators of irreducible representations of the quantum resonance algebras $\mathfrak{F}_{\omega}=\mathfrak{F}_{l, m}$ have to be differential operators, not pseudodifferential (the maximal order of these operators is $\max (l, m)$ ).

Thus the geometry [26, 27] determining the Wick-Klauder-Berezin *product on the gyron phase space has a purely quantum behavior and the *-product itself cannot be obtained by a formal deformation technique from the classical data.

Note that here we mean the phase spaces corresponding either to low energy levels of the oscillator (i.e., to the nano-zone near its equilibrium point, in the terminology of [24]) or to excited levels (i.e., to the micro-zone). Thus one can talk about quantum nano- or micro-geometry generated by the $l: m$ frequency resonance.

The distinction between the specific case $l=m=1$ and the generic case $\max (l, m)>1$ is the distinction between algebras with linear and nonlinear commutation relations. We see that the nonlinearity of relations in the algebra $\mathfrak{F}_{l, m}$ (the absence of a Lie group of symmetries) for the resonance oscillator implies the quantum character of the phase spaces in nano- and micro-zones near the ground state. The motion in these spaces is the gyron dynamics. In the nano-zone, this dynamics is purely quantum and does not have a classical analog at all. In the micro-zone, the gyron dynamics and the gyron spectrum can be described by semiclassical methods [23, 24] if one at first fixes the quantum geometry of the gyron phase space.

Applying this theory, for instance, to optical gyrons, we come to the conclusion that the light beam propagating near the axis of a resonance fiber waveguide cannot be described by purely geometric optics and carry essentially quantum properties. This opens an opportunity to apply such simple optical devices, for example, in constructing elements of quantum computers.

Also note that the $l: m$ resonance oscillators, which we discuss here, can be presented in the form

$$
\hat{l}+\hat{m}
$$


where $\hat{l}$ and $\hat{m}$ are mutually commutating action operators with spectra $l \cdot \mathbb{Z}_{+}$ and $m \cdot \mathbb{Z}_{+}$in the Hilbert space $\mathcal{L}=L^{2}(\mathbb{R} \times \mathbb{R})$. The operators $\hat{l}$ and $\hat{m}$ can be considered as "quantum integer numbers" and their sum as a quantum sum of integers. Then the representation theory of the algebra $\mathfrak{F}_{l, m}$ and the corresponding quantum geometry could be considered as a brick to construction of something like "quantum arithmetics."

\section{Commutation relations and Poisson brack- ets for $l: m$ resonance}

The Hamiltonian of the resonance oscillator (1.3) can be written as

$$
\mathbf{E}=l \mathbf{b}_{1}^{*} \mathbf{b}_{1}+m \mathbf{b}_{2}^{*} \mathbf{b}_{2} .
$$

Here $l, m$ are coprime integers, $\mathbf{b}_{1}, \mathbf{b}_{2}$ are annihilation operators in the Hilbert space $\mathcal{L}$, and $\mathbf{b}_{1}^{*}, \mathbf{b}_{2}^{*}$ are the conjugate creation operators. The commutation relations are

$$
\left[\mathbf{b}_{1}, \mathbf{b}_{1}^{*}\right]=\left[\mathbf{b}_{2}, \mathbf{b}_{2}^{*}\right]=\hbar \mathbf{I},
$$

all other commutators are zero.

In the algebra generated by $\mathbf{b}_{1}, \mathbf{b}_{2}, \mathbf{b}_{1}^{*}, \mathbf{b}_{2}^{*}$, let us consider the commutant of the element (2.1). This commutant is a nontrivial, noncommutative subalgebra. We call it a resonance algebra. It is related to quantum gyrons.

Note that the resonance algebra is generated by the following four elements:

$$
\mathbf{A}_{1}=\mathbf{b}_{1}^{*} \mathbf{b}_{1}, \quad \mathbf{A}_{2}=\mathbf{b}_{2}^{*} \mathbf{b}_{2}, \quad \mathbf{A}_{+}=\left(\mathbf{b}_{2}^{*}\right)^{l} \mathbf{b}_{1}^{m}, \quad \mathbf{A}_{-}=\mathbf{A}_{+}^{*} .
$$

Let us define the following polynomials

$$
\begin{aligned}
& \rho\left(A_{1}, A_{2}\right) \stackrel{\text { def }}{=} \prod_{j=1}^{m}\left(A_{1}+j \hbar\right) \cdot \prod_{s=1}^{l}\left(A_{2}-s \hbar+\hbar\right), \\
& \varkappa\left(A_{1}, A_{2}\right) \stackrel{\text { def }}{=} l A_{1}+m A_{2} .
\end{aligned}
$$

Lemma 2.1. Elements (2.2) obey the commutation relations

$$
\begin{gathered}
{\left[\mathbf{A}_{1}, \mathbf{A}_{2}\right]=0} \\
{\left[\mathbf{A}_{1}, \mathbf{A}_{ \pm}\right]=\mp \hbar m \mathbf{A}_{ \pm}, \quad\left[\mathbf{A}_{2}, \mathbf{A}_{ \pm}\right]= \pm \hbar l \mathbf{A}_{ \pm}} \\
{\left[\mathbf{A}_{-}, \mathbf{A}_{+}\right]=\rho\left(\mathbf{A}_{1}-\hbar m, \mathbf{A}_{2}+\hbar l\right)-\rho\left(\mathbf{A}_{1}, \mathbf{A}_{2}\right) .}
\end{gathered}
$$


Lemma 2.2. In the abstract algebra $\mathfrak{F}_{l, m}$ with relations (2.4) there are two Casimir elements

$$
\boldsymbol{\kappa}=\varkappa\left(\mathbf{A}_{1}, \mathbf{A}_{2}\right), \quad \mathbf{C}=\mathbf{A}_{+} \mathbf{A}_{-}-\rho\left(\mathbf{A}_{1}, \mathbf{A}_{2}\right) .
$$

In realization (2.2) the Casimir element $\mathbf{C}$ is identically zero, and the Casimir element $\boldsymbol{\kappa}$ coincides with the oscillator Hamiltonian $\mathbf{E}(2.1)$.

Note that the operators $\mathbf{A}_{1}, \mathbf{A}_{2}(2.2)$ are self-adjoint, but $\mathbf{A}_{+}$is not. Let us introduce the self-adjoint operators $\mathbf{A}_{3}, \mathbf{A}_{4}$ by means of the equalities

$$
\mathbf{A}_{ \pm}=\mathbf{A}_{3} \mp i \mathbf{A}_{4} .
$$

Then commutation relations (2.4) read

$$
\begin{gathered}
{\left[\mathbf{A}_{1}, \mathbf{A}_{2}\right]=0, \quad\left[\mathbf{A}_{1}, \mathbf{A}_{3}\right]=i \hbar m \mathbf{A}_{4}, \quad\left[\mathbf{A}_{1}, \mathbf{A}_{4}\right]=-i \hbar m \mathbf{A}_{3},} \\
{\left[\mathbf{A}_{2}, \mathbf{A}_{3}\right]=-i \hbar l \mathbf{A}_{4}, \quad\left[\mathbf{A}_{2}, \mathbf{A}_{4}\right]=i \hbar l \mathbf{A}_{3},} \\
{\left[\mathbf{A}_{3}, \mathbf{A}_{4}\right]=\frac{i}{2}\left(\rho\left(\mathbf{A}_{1}-\hbar m, \mathbf{A}_{2}+\hbar l\right)-\rho\left(\mathbf{A}_{1}, \mathbf{A}_{2}\right)\right) .}
\end{gathered}
$$

Let us denote by $A_{j}$ the classical variable (a coordinate on $\mathbb{R}^{4}$ ) corresponding to the quantum operator $\mathbf{A}_{j}$. Then the relations (2.4a) are reduced to the following Poisson brackets on $\mathbb{R}^{4}$ :

$$
\begin{aligned}
& \left\{A_{1}, A_{2}\right\}=0, \\
& \left\{A_{1}, A_{3}\right\}=-m A_{4}, \quad\left\{A_{1}, A_{4}\right\}=m A_{3}, \\
& \left\{A_{2}, A_{3}\right\}=l A_{4}, \quad\left\{A_{2}, A_{4}\right\}=-l A_{3}, \\
& \left\{A_{4}, A_{3}\right\}=\frac{1}{2}\left(l^{2} A_{1}-m^{2} A_{2}\right) A_{1}^{m-1} A_{2}^{l-1} .
\end{aligned}
$$

Lemma 2.3. Relations (2.5) determine the Poisson brackets on $\mathbb{R}^{4}$ with the Casimir functions

$$
\varkappa=l A_{1}+m A_{2}, \quad C=A_{3}^{2}+A_{4}^{2}-A_{1}^{m} A_{2}^{l} .
$$

Lemma 2.4. In the subset in $\mathbb{R}^{4}$ determined by the inequalities $A_{1} \geq 0$ and $A_{2} \geq 0$, there is a family of surfaces

$$
\Omega=\{\varkappa=E, C=0\}, \quad E>0,
$$

which coincide with the closure of symplectic leaves $\Omega_{0}$ of the Poisson structure (2.5). These surfaces are diffeomorphic to the sphere: $\Omega \approx \mathbb{S}^{2}$.

The topology of the symplectic leaves $\Omega_{0}$ is the following: 
- if $l=m=1$, then $\Omega_{0}=\Omega$;

- if $l=1, m>1$ or $l>1, m=1$, then $\Omega_{0}$ is obtained from $\Omega$ by deleting the point $\left(0, \frac{E}{m}, 0,0\right)$ or the point $\left(\frac{E}{l}, 0,0,0\right)$;

- if $l>1, m>1$, then $\Omega_{0}$ is obtained from $\Omega$ by deleting both the points $\left(0, \frac{E}{m}, 0,0\right)$ and $\left(\frac{E}{l}, 0,0,0\right)$.

Lemma 2.5. If $l>1$ or $m>1$, then the Kirillov symplectic form $\omega_{0}$ on the leaf $\Omega_{0} \subset \Omega$ has a weak (integrable) singularity at the point $A_{2}=0$ or $A_{1}=0$. The symplectic volume of $\Omega_{0}$ is finite

$$
\frac{1}{2 \pi} \int_{\Omega_{0}} \omega_{0}=\frac{E}{l m}
$$

Lemma 2.6. On the subset $A_{1}>0$ the complex coordinate

$$
z_{0}=\frac{A_{3}+i A_{4}}{A_{1}^{m}}
$$

determines a partial complex structure consistent with the brackets (2.5) in the sense of [32. On each symplectic leave $\Omega_{0}$, this partial complex structure generates the Kählerian structure with the potential

$$
\Phi_{0}=\int_{0}^{\left|z_{0}\right|^{2}}\left(\frac{E}{2 l m}+\alpha_{E}(x)\right) \frac{d x}{x}, \quad \omega_{0}=i \bar{\partial} \partial \Phi_{0} .
$$

Here $\partial$ is the differential by $z_{0}$ and $\alpha_{E}=\alpha_{E}(x)$ is the solution of the equation

$$
x=\left(\frac{E}{2 m}+l \alpha_{E}\right)^{l}\left(\frac{E}{2 l}-m \alpha_{E}\right)^{-m}
$$

with values on the interval $-\frac{E}{2 l m} \leq \alpha_{E} \leq \frac{E}{2 l m}$.

The singular points of $\omega_{0}$ on $\Omega_{0}$ correspond to the poles

$$
\begin{aligned}
& A_{2}=0 \quad \Longleftrightarrow \quad z_{0}=0, \quad \omega_{0} \sim \frac{1}{l^{2}}\left(\frac{E}{l}\right)^{m / l} \frac{d x \wedge d \varphi}{x^{1-1 / l}} \quad \text { as } \quad z_{0} \rightarrow 0, \\
& A_{1}=0 \quad \Longleftrightarrow \quad z_{0}=\infty, \quad \omega_{0} \sim \frac{1}{m^{2}}\left(\frac{E}{m}\right)^{l / m} \frac{d x \wedge d \varphi}{x^{1+1 / m}} \quad \text { as } \quad z_{0} \rightarrow \infty,
\end{aligned}
$$


where $(x, \varphi)$ are polar coordinates, $z_{0}=x^{1 / 2} \exp \{i \varphi\}$.

The restrictions of coordinate functions to the surface (2.6) are given by

$$
\begin{gathered}
\left.A_{1}\right|_{\Omega_{0}}=\frac{E}{2 l}-m \alpha_{E}\left(\left|z_{0}\right|^{2}\right),\left.\quad A_{2}\right|_{\Omega_{0}}=\frac{E}{2 m}+l \alpha_{E}\left(\left|z_{0}\right|^{2}\right), \\
\left.\left(A_{3}+i A_{4}\right)\right|_{\Omega_{0}}=z_{0}\left(\frac{E}{2 l}-m \alpha_{E}\left(\left|z_{0}\right|^{2}\right)\right)^{m} .
\end{gathered}
$$

Note that the properties of classical symplectic leaves of the $l: m$ resonance algebra, described in Lemmas 2.4-2.6, are a particular case of the topology and geometry of toric varieties (in our case the torus $\mathbb{T}^{1}=\mathbb{S}^{1}$ is the cycle); about this see general theorems in [28]-30]. The Poisson extension (2.5) by means of polynomial brackets was first described in 22, 23] for the case of $1: 2$ resonance and in 24, 25] for the $l: m$ case, as well for the general multidimensional resonances. A type of Poisson extension was also considered in 31] for some specific class of resonance proportions (which does not include, for instance, the $1: 2: 3$ resonance).

\section{Irreducible representations of $l: m$ reso- nance algebra}

First of all, let us discuss the basic problems in constructing irreducible representations of algebras like (2.4), (2.4a). Following the standard geometric quantization program [6] one has to choose a line bundle over symplectic leaves $\Omega_{0}$ of the Poisson algebra related to $(2.4 \mathrm{a})$, that is, the Poisson algebra (2.5). Then this bundle is endowed with the Hermitian connection whose curvature is $i \omega_{0}$, and a Hilbert space $\mathcal{H}_{0}$ of antiholomorphic sections of the bundle is introduced. In this Hilbert space, the operators of irreducible representation of the algebra (2.4a) are supposed to act and to be self-adjoint.

However, there are two principle difficulties. First, we do not know which measure on $\Omega_{0}$ to take in order to determine the Hilbert norm in the space $\mathcal{H}_{0}$. The choice of measure should imply the reproducing property [32, 33]

$$
\omega_{0}=i \bar{\partial} \partial \ln \sum_{k}\left|\varphi_{0}^{(k)}\right|^{2}
$$

where $\left\{\varphi_{0}^{(k)}\right\}$ is an orthonormal basis in $\mathcal{H}_{0}$. For the inhomogeneous case, where the commutation relations (2.4a) are not linear and no Lie group acts 
on $\Omega_{0}$, the existence of such a reproducing measure is, in general, unknown. This difficulty was discovered in 34] (more precisely, it was observed in 34] that the Liouville measure generated by the symplectic form $\omega_{0}$ does not obey the property (3.1) in general).

Secondly, even if one knows the reproducing measure, there is still a problem: the operators of the irreducible representation constructed canonically by the geometric quantization scheme would be pseudodifferential, but not differential operators. There are additional nontrivial conditions on the complex structure (polarization) that make the generators of the algebra be differential operators (of order greater than 1, in general). About such highest analogs of the Blattner-Kostant-Sternberg conditions for the polarization to be "invariant" see in [35, 36].

Taking these difficulties into account, we modify the quantization scheme. From the very beginning, we look for an appropriate complex structure and the scalar product in the space of antiholomorphic functions that guarantee the existence of an Hermitian representation of the given algebra by differential operators, and then introduce a "quantum" Kählerian form $\omega$ on $\Omega$, a "quantum" measure and the "quantum" Hilbert space $\mathcal{H}$ which automatically obeys the reproducing property like (3.1) (without "classical" label 0). This approach is explained in [32, 33, 37].

Note that the polynomial structure of the right-hand sides of relations (2.4), (2.4a) is critically important in this scheme to obtain representations by differential operators.

Denote by $\mathcal{P}_{r}$ the space of all polynomials $\varphi(\lambda)=\sum_{n=0}^{r} \varphi_{n} \lambda^{n}$ of degree $r \geq 0$ with complex coefficients.

Lemma 3.1. Let $f_{+}, f_{-}$be two complex functions on $\mathbb{Z}_{+}$such that

$$
\begin{aligned}
& f_{+} f_{-}>0 \quad \text { on the subset }\{1, \ldots, r\} \subset \mathbb{Z}_{+}, \\
& f_{-}(0)=f_{+}(r+1)=0 .
\end{aligned}
$$

Then the differential operators

$$
\mathbf{a}_{+}=f_{+}\left(\lambda \frac{d}{d \lambda}\right) \cdot \lambda, \quad \mathbf{a}_{-}=\frac{1}{\lambda} \cdot f_{-}\left(\lambda \frac{d}{d \lambda}\right)
$$

leave the space $\mathcal{P}_{r}$ invariant and they are conjugate to each other with respect to the following scalar product in $\mathcal{P}_{r}$ :

$$
\left(g, g^{\prime}\right) \stackrel{\text { def }}{=} \sum_{n=0}^{r} \prod_{s=1}^{n} \overline{\frac{f_{-}(s)}{f_{+}(s)}} \varphi_{n} \overline{\varphi_{n}^{\prime}} .
$$


Any operator $f\left(\lambda \frac{d}{d \lambda}\right)$, where $f$ is a real function on $\mathbb{Z}_{+}$, is self-adjoint in $\mathcal{P}_{r}$ with respect to this scalar product.

Now we consider a map

$$
\gamma: \mathbb{R}^{k} \rightarrow \mathbb{R}^{k}
$$

and a real function $\rho$ on $\mathbb{R}^{k}$. Denote by $R_{r} \subset \mathbb{R}^{k}$ the subset of all points $a_{0}$ such that

$$
\begin{aligned}
\rho\left(\gamma^{r+1}\left(a_{0}\right)\right) & =\rho\left(a_{0}\right), \\
\rho\left(\gamma^{n}\left(a_{0}\right)\right) & >\rho\left(a_{0}\right) \quad(n=1, \ldots, r) .
\end{aligned}
$$

For any $a_{0} \in R_{r}$ we define real functions $f_{j}(j=1, \ldots, k)$ on $\mathbb{Z}_{+}$by the formula $f_{j}(n) \stackrel{\text { def }}{=} \gamma^{n}\left(a_{0}\right)_{j}$, and introduce mutually commuting operators in the space $\mathcal{P}_{r}$ :

$$
\mathbf{a}_{j} \stackrel{\text { def }}{=} f_{j}\left(\lambda \frac{d}{d \lambda}\right) .
$$

Lemma 3.2. Let $a_{0} \in R_{r}$, and let there be a factorization

$$
\rho\left(\gamma^{n}\left(a_{0}\right)\right)-\rho\left(a_{0}\right)=f_{+}(n) f_{-}(n), \quad 0 \leq n \leq r+1,
$$

where the factors $f_{ \pm}$obey the property (3.2). Then the operator $\mathbf{a}_{+}$(3.3) and $\mathbf{a}_{j}$ (3.6) in the space $\mathcal{P}_{r}$ with the scalar product (3.4) satisfy the relations

$$
\mathbf{a}_{+}^{*}=\mathbf{a}_{-}, \quad \mathbf{a}_{j}^{*}=\mathbf{a}_{j} \quad(j=1, \ldots, k),
$$

and

$$
\begin{aligned}
& {\left[\mathbf{a}_{j}, \mathbf{a}_{s}\right]=0,} \\
& \mathbf{a}_{j} \mathbf{a}_{+}=\mathbf{a}_{+} \gamma_{j}(\mathbf{a}), \quad \mathbf{a}_{-} \mathbf{a}_{j}=\gamma_{j}(\mathbf{a}) \mathbf{a}_{-} \quad(j=1, \ldots, k), \\
& {\left[\mathbf{a}_{-}, \mathbf{a}_{+}\right]=\rho(\gamma(\mathbf{a}))-\rho(\mathbf{a}) .}
\end{aligned}
$$

Lemma 3.3. Consider the abstract algebra $\mathfrak{F}$ with relations (3.8). The element $\mathbf{C}=\mathbf{a}_{+} \mathbf{a}_{-}-\rho(\mathbf{a})$ belongs to the center of $\mathfrak{F}$. If a function $\varkappa$ on $\mathbb{R}^{k}$ is $\gamma$-invariant, then the element $\boldsymbol{\kappa}=\varkappa(\mathbf{a})$ belongs to the center of $\mathfrak{F}$.

In the representation (3.3), (3.6), these central elements are scalar: $\mathbf{C}=$ $\rho\left(a_{0}\right) \cdot \mathbf{I}, \boldsymbol{\kappa}=\varkappa\left(a_{0}\right) \cdot \mathbf{I}$. This representation of the algebra $\mathfrak{F}$ is irreducible and Hermitian.

If the map $\gamma$ has no fixed points, then all irreducible Hermitian representations of the algebra $\mathfrak{F}$ can be obtained in this way. All such representations of dimension $r+1$ are parameterized by elements of the set $R_{r}(r=0,1,2, \ldots)$. 
Now let us return to commutation relations (2.4). In this case $k=2$, the function $\rho$ is given by (2.3), and the mapping $\gamma \equiv \Gamma^{\hbar}: \mathbb{R}^{2} \rightarrow \mathbb{R}^{2}$ is

$$
\Gamma^{\hbar}\left(\begin{array}{c}
A_{1} \\
A_{2}
\end{array}\right) \stackrel{\text { def }}{=}\left(\begin{array}{c}
A_{1}-\hbar m \\
A_{2}+\hbar l
\end{array}\right) .
$$

It follows from (2.2) that we have to be interested in a subset $A_{1} \geq 0$, $A_{2} \geq 0$ in $\mathbb{R}^{2}$. Also in view of Lemma 2.2, the values of the Casimir element $\mathbf{C}=\rho\left(a_{0}\right) \cdot \mathbf{I}$ must be zero. From (3.5) we obtain

$$
\begin{aligned}
& \rho\left(a_{0}\right)=\rho\left(\Gamma^{\hbar(r+1)}\left(a_{0}\right)\right)=0, \\
& \rho\left(\Gamma^{\hbar n}\left(a_{0}\right)\right)>0 \quad(n=1, \ldots, r) .
\end{aligned}
$$

Using (2.3) let us factorize:

$$
\rho=\rho_{+} \rho_{-}, \quad \rho_{+}(A) \stackrel{\text { def }}{=} \prod_{j=1}^{m}\left(A_{1}+\hbar j\right), \quad \rho_{-}(A) \stackrel{\text { def }}{=} \prod_{s=1}^{l}\left(A_{2}-\hbar s+\hbar\right) .
$$

It is possible to satisfy (3.7) by choosing

$$
f_{ \pm}(n)=\rho_{ \pm}\left(\Gamma^{\hbar n}\left(a_{0}\right)\right) .
$$

In this case, the set $R_{r} \subset \mathbb{R}^{2}$ consists of all points $a_{0}=\left(\begin{array}{c}\hbar(r m+p) \\ \hbar q\end{array}\right)$ for which the pair of integers $p, q$ obeys the inequalities

$$
0 \leq q \leq l-1, \quad 0 \leq p \leq m-1
$$

The $\gamma$-invariant function $\varkappa$ in our case (3.9) is just $\varkappa(A)=l A_{1}+m A_{2}$. In view of Lemma 3.3, the value of the second Casimir element $\boldsymbol{\kappa}=\varkappa(\mathbf{a})$ in the irreducible representation (3.3), (3.6) is $\varkappa\left(a_{0}\right)=E_{r, q, p}$, where

$$
E_{r, q, p} \stackrel{\text { def }}{=} \hbar(l m r+l p+m q) \text {. }
$$

From Lemma 2.2 we conclude that these numbers coincide with eigenvalues of the oscillator $\mathbf{E}(2.1)$.

Also from (3.4) we see that the scalar product in the space $\mathcal{P}_{r}$ is given by

$$
\left(\varphi, \varphi^{\prime}\right)=\sum_{n=0}^{r} \hbar^{(l-m) n} \frac{(q+n l) !(p+(r-n) m) !}{q !(p+r m) !} \varphi_{n} \overline{\varphi_{n}^{\prime}}
$$


Thus the vector space of the irreducible representation depends on the number $r$ only, but its Hilbert structures are parameterized by the pairs $q, p$ from (3.11). That is why below we will use the notation $\mathcal{P}_{r} \equiv \mathcal{P}_{r, q, p}$.

Let us summarize the obtained results.

Theorem 3.4. The commutant of the $l: m$ resonance oscillator $\mathbf{E}(2.1)$ is generated by operators (2.2) obeying commutation relation (2.4). The irreducible representation of the algebra (2.4), corresponding to the eigenvalue $E_{r, q, p}(3.12)$ of the operator $\mathbf{E}$, is given by the following ordinary differential operators $\mathbf{a}=\left(\mathbf{a}_{1}, \mathbf{a}_{2}\right)$ and $\mathbf{a}_{ \pm}$:

$$
\mathbf{a}=\Gamma^{\hbar \lambda \frac{d}{d \lambda}}\left(a_{0}\right), \quad \mathbf{a}_{+}=\rho_{+}(\mathbf{a}) \cdot \lambda, \quad \mathbf{a}_{-}=\frac{1}{\lambda} \cdot \rho_{-}(\mathbf{a}) .
$$

Here $a_{0}=\left(\begin{array}{c}\hbar(r m+p) \\ \hbar q\end{array}\right)$, the flow $\Gamma$ on $\mathbb{R}^{2}$ is defined by (3.9) and the factors $\rho_{ \pm}$are defined by (3.10). The representation (3.14) acts in the space $\mathcal{P}_{r, q, p}$ of polynomials in $\lambda$ of degree $r$, and it is Hermitian with respect to the scalar product (3.13). The dimension of this representation is $r+1$.

In fact, formula (3.14) determines just the matrix representations of the algebra (2.4): elements $\mathbf{a}$ are represented by a diagonal matrix and $\mathbf{a}_{ \pm}$by near-diagonal matrices with respect to the orthonormal basis of monomials

$$
\varphi^{(k)}(\lambda)=\hbar^{(m-l) k / 2}\left(\frac{q !(p+r m) !}{(q+k l) !(p+(r-k) m) !}\right)^{1 / 2} \cdot \lambda^{k} \quad(k=0, \ldots, r)
$$

in the space $\mathcal{P}_{r, q, p}$. These matrices are real-valued and determined by the integer numbers $l, m$ (from the resonance proportion) and $r, p, q$ (labeling the representation):

$$
\begin{aligned}
& \left(\mathbf{a}_{1}\right)_{n s}=\hbar(p+(r-n) m) \delta_{n, s}, \quad\left(\mathbf{a}_{2}\right)_{n s}=\hbar(q+n l) \delta_{n, s}, \\
& \left(\mathbf{a}_{+}\right)_{n s}=\hbar^{(l+m) / 2}\left(\frac{(q+n l) !(p+(r-s) m) !}{(q+s l) !(p+(r-n) m) !}\right)^{1 / 2} \delta_{n-1, s}, \\
& \left(\mathbf{a}_{-}\right)_{n s}=\left(\mathbf{a}_{+}\right)_{s n} .
\end{aligned}
$$

Here the matrix indices $n, s$ run over the set $\{0, \ldots, r\}$ and $\delta_{n, s}$ are the Kronecker symbols.

In the particular case $l=m=1$, from (3.16) one obtains the well-known Hermitian matrix irreducible representations of the "spin" Lie algebra $\mathrm{su}(2)$ with cyclic commutation relation between generators $\frac{1}{2}\left(\mathbf{A}_{1}-\mathbf{A}_{2}\right), \frac{1}{2}\left(\mathbf{A}_{+}+\right.$ $\left.\mathbf{A}_{-}\right), \frac{i}{2}\left(\mathbf{A}_{+}-\mathbf{A}_{-}\right)$. 


\section{Quantum geometry of the $l: m$ resonance}

Now we give a geometric interpretation of the obtained representations of the resonance algebra.

It follows from (3.4) that the element $\rho_{+}(\mathbf{A})^{-1}\left(\mathbf{A}_{3}-i \mathbf{A}_{4}\right)$, in the algebra generated by relations (2.4), is represented by the multiplication by $\lambda$ in each irreducible representation (3.14). If we denote

$$
\mathbf{z}=\left(\mathbf{A}_{3}+i \mathbf{A}_{4}\right) \rho_{+}(\mathbf{A})^{-1},
$$

then the conjugate operator $\mathbf{z}^{*}$ in each irreducible representation can be taken equal to the multiplication by a complex variable $\bar{z}$ :

$$
\mathbf{z}^{*}=\bar{z}
$$

Thus, here we change our notation and use $\bar{z}$ instead of $\lambda$. From now on, $\mathcal{P}_{r, q, p}$ is the space of anti-holomorphic functions (polynomials in $\bar{z}$ of degree $\leq r)$ on $\mathbb{R}^{2}$.

Let us assume that the scalar product (3.13) in the space $\mathcal{P}_{r, q, p}$ can be written in the integral form

$$
\left(\varphi, \varphi^{\prime}\right)=\frac{1}{2 \pi \hbar} \int_{\mathbb{R}^{2}} \varphi(\bar{z}(a)) \overline{\varphi^{\prime}(\bar{z}(a))} L(a) d a,
$$

where $d a=|d \bar{z}(a) \wedge d z(a)|$ and $a \rightarrow z(a)$ is the complex coordinate on $\mathbb{R}^{2}$.

Lemma 4.1. The explicit formula for the density $L$ in (4.2) is

$L(a)=\frac{1}{4 \hbar^{r m+p+q+1}(p+r m) ! q ! x} \int_{0}^{\infty} A_{1}^{r m+p} A_{2}^{q}\left(\frac{l^{2}}{A_{2}}+\frac{m^{2}}{A_{1}}\right)^{-1} \exp \left\{-\frac{A_{1}+A_{2}}{2 \hbar}\right\} d E$,

where $A_{1}=\frac{E}{2 l}-m \alpha_{E}(x), A_{2}=\frac{E}{2 m}+l \alpha_{E}(x), \alpha_{E}$ is taken from (2.10), and $x=|z(a)|^{2}$.

These are first steps to assign some geometry to the quantum algebra (2.4) and its irreducible representations. The next step is to consider the multiplication operation in this algebra.

Note that linear operators in $\mathcal{P}_{r, q, p}$ can be presented by their kernels. So, the algebra of operators is naturally isomorphic to $\mathcal{S}_{r, q, p} \stackrel{\text { def }}{=} \mathcal{P}_{r, q, p} \otimes \overline{\mathcal{P}}_{r, q, p}$. The operator product is presented by the convolution of kernels which is 
generated by pairing between $\overline{\mathcal{P}}_{r, q, p}$ and $\mathcal{P}_{r, q, p}$ given by the scalar product (3.13).

The algebra $\mathcal{S}_{r, q, p}$ consists of functions in $\bar{z}, z$, they are polynomials on $\mathbb{R}^{2}$. On this function space we have a noncommutative product (convolution), but the unity element of this convolution is presented by the function

$$
K=\sum_{k=0}^{r} \varphi^{(k)} \otimes \overline{\varphi^{(k)}}
$$

where $\varphi^{(k)}$ is the orthonormal basis in $\mathcal{P}_{r, q, p}$. This function is called a reproducing kernel [38, 39], it is independent of the choice of the basis $\left\{\varphi^{(k)}\right\}$. From (3.15) we see the explicit formula for the reproducing kernel

$$
K=k\left(|z|^{2}\right), \quad k(x) \stackrel{\text { def }}{=} \sum_{n=0}^{r} \hbar^{(m-l) n} \frac{q !(p+r m) !}{(q+n l) !(p+(r-n) m) !} x^{n} .
$$

In order to give a Gelfand type spectral-geometric interpretation of some algebra, we, first of all, have to ensure that the unity element of this algebra is presented by the unity function. It is not so for the algebra $\mathcal{S}_{r, q, p}$. That is why we have to divide the "kernel elements" from $\mathcal{S}_{r, q, p}$ by the reproducing kernel (4.4). The correct function algebra consists of ratios of the type

$$
f=\frac{\varphi \otimes \overline{\varphi^{\prime}}}{K}
$$

where $\varphi, \varphi^{\prime} \in \mathcal{P}_{r, q, p}$. The product of two functions of this type generated by the convolution of kernels is given by

$$
\left(f_{1} * f_{2}\right)(a)=\frac{1}{2 \pi \hbar} \int_{\text {phase space }} f_{1}^{\#}(a \mid b) f_{2}^{\#}(b \mid a) p_{a}(b) d m(b) .
$$

Here

$$
\begin{aligned}
d m(b) & \stackrel{\text { def }}{=} L(b) K(b) d b, \\
p_{a}(b) & \stackrel{\text { def }}{=}\left|K^{\#}(a \mid b)\right|^{2} K(a)^{-1} K(b)^{-1},
\end{aligned}
$$

and the operation $f \rightarrow f^{\#}$ denotes the analytic continuation holomorphic with respect to the "right" argument and anti-holomorphic with respect to the "left" argument in the notation $f^{\#}(\cdot \mid \cdot)$. The product (4.6) possesses the desirable property: $1 * f=f * 1=f$. 
Let us look at formula (4.5). Since $f$ is going to be a function on an invariant geometric space, $\varphi$ and $\varphi^{\prime}$ have to be sections of a Hermitian line bundle with the curvature form

$$
\omega=i \hbar \bar{\partial} \partial \ln K \equiv i g d \bar{z} \wedge d z .
$$

Here $\partial$ denotes the differential by $z$. Formula (4.9) means that the measure $d m$ (4.7) is the reproducing measure with respect to the Kählerian form $\omega$ in the sense [33.

Note that formula (4.9) defines both the quantum form $\omega$ and the quantum metric $g=g\left(|z|^{2}\right), g(x)=\hbar \frac{d}{d x}\left(x \frac{d}{d x}(\ln k(x))\right)$ via the polynomial (4.4).

After the quantum form $\omega$ appears, the "probability" factor $p_{a}$ in the noncommutative product (4.6) can be written as

$$
p_{a}(b)=\exp \left\{\frac{i}{\hbar} \int_{\sum(a, b)} \omega\right\} .
$$

Here $\sum(a, b)$ is a membrane in the complexified space whose boundary consists of four paths connecting points $a \rightarrow b|a \rightarrow b \rightarrow a| b \rightarrow a$ along leaves of the complex polarization and its conjugate [26, 40].

Note that the set of functions (4.10) makes up a resolution of unity:

$$
\frac{1}{2 \pi \hbar} \int_{\text {phase space }} p_{a} d m(a)=1,
$$

and each $p_{a}$ is the "eigenfunction" of the operators of left or right multiplication:

$$
f * p_{a}=f(\cdot \mid a) p_{a}, \quad p_{a} * f=f(a \mid \cdot) p_{a} .
$$

The details about such a way to establish a correspondence between quantum algebras and Kählerian geometry can be found in 33.

Let us discuss global aspects of this quantum geometry. The Kählerian form $\omega$ (4.9) is actually well defined on the compactified plane $\mathbb{R}^{2} \cup\{\infty\}$ which includes the infinity point $z=\infty$. To see this, we just can make the change of variables $z^{\prime}=1 / z$ and observe that $\omega$ is smooth near $z^{\prime}=0$.

Thus the actual phase space is diffeomorphic to $\mathbb{S}^{2}$ and we have

$$
\frac{1}{2 \pi \hbar} \int_{\mathbb{S}^{2}} \omega=r, \quad \frac{1}{2 \pi \hbar} \int_{\mathbb{S}^{2}} d m=r+1 .
$$

The first formula (4.13) follows from the fact that $K \sim$ const $\cdot|z|^{2 r}$ as $z \rightarrow$ $\infty$ (see in (4.4)). It means that the cohomology class $\frac{1}{2 \pi \hbar}[\omega]$ is integer, and 
this is the necessary condition for the Hermitian bundle with the curvature $i \omega$ over $\mathbb{S}^{2}$ to have global sections [41].

The second formula (4.13) follows from the definition (4.3) which implies $\frac{1}{2 \pi \hbar} \int d m=\sum_{k=0}^{r}\left\|\varphi^{(k)}\right\|^{2}$, where the norm of each $\varphi^{(k)}$ is taken in the sense (4.3) and is equal to 1 by definition. The number $r+1$ in (4.13) is the dimension of the irreducible representation of the resonance algebra.

We stress that the quantum Kählerian form $\omega$, given by (4.4), (4.9), and the quantum measure $d m$, given by (4.7) and Lemma 4.1, are essentially different from the classical form $\omega_{0}(2.9)$ and the classical Liouville measure $d m_{0}=\left|\omega_{0}\right|$. The main difference is that $\omega$ is smooth and $d m$ is regular at poles while $\omega_{0}$ and $d m_{0}$ are not. Some information regarding asymptotics of the quantum objects as $\hbar \rightarrow 0$ and asymptotics near the poles is summarized in the following lemma.

Lemma 4.2. (a) In the classical limit $\hbar \rightarrow 0, E_{r, q, p} \rightarrow E>0$, out of neighborhoods of the poles $z=0$ and $z=\infty$ on the sphere, the quantum geometrical objects are approximated by the classical ones:

$$
\omega=\omega_{0}+O(\hbar), \quad d m=d m_{0}(1+O(\hbar)) .
$$

(b) The behavior of the quantum reproducing measure near the poles is the following:

$$
\begin{aligned}
& d m \sim \operatorname{const} \cdot \frac{d x \wedge d \varphi}{x^{1-(q+1) / l}} \quad \text { as } \quad x \rightarrow 0, \\
& d m \sim \operatorname{const} \cdot \frac{d x \wedge d \varphi}{x^{1+(p+1) / m}} \quad \text { as } \quad x \rightarrow \infty,
\end{aligned}
$$

where $z=x^{1 / 2} \exp \{i \varphi\}$. Thus the reproducing measure has weak singularities at poles.

(c) Near the poles, the quantum Kählerian form looks as

$$
\begin{aligned}
& \omega \sim \hbar^{m-l+1} \frac{(p+r m) ! q !}{(p+r m-m) !(q+l) !} i d \bar{z} \wedge d z \quad \text { as } \quad z \rightarrow 0, \\
& \omega \sim \hbar^{l-m+1} \frac{p !(q+r l) !}{(p+m) !(q+r l-l) !} \frac{i d \bar{z} \wedge d z}{|z|^{4}} \quad \text { as } \quad z \rightarrow \infty .
\end{aligned}
$$

Thus, near the poles, the asymptotics of $\omega$ as $\hbar \rightarrow 0$ is

$$
\begin{aligned}
& \omega \sim \text { const } \hbar^{1-l} i d \bar{z} \wedge d z \quad(z \sim 0), \\
& \omega \sim \text { const } \hbar^{1-m} \frac{i d \bar{z} \wedge d z}{|z|^{4}} \quad(z \sim \infty) .
\end{aligned}
$$


Comparing (4.14) with (2.11) we see that, near poles, $d m$ is not approximated by $d m_{0}$ as $\hbar \rightarrow 0$ if $q>0$ or $p>0$. So, the usual deformation theory (starting with classical data) cannot be applied to compute the reproducing measure globally on the phase space.

Formulas (4.15) demonstrate that the quantum $\omega$ is not approximated by $\omega_{0}$ as $\hbar \rightarrow 0$ near the poles; the classical form $\omega_{0}$ must be singular at $z=0$ if $l>1$ and be singular at $z=\infty$ if $m>1$. This statement is in agreement with (2.11).

Note that the cohomology class of the classical symplectic form $\omega_{0}$ on the classical leaf with the quantized energy $E=E_{r, q, p}$ (3.12) is given by (2.7):

$$
\frac{1}{2 \pi \hbar} \int_{\Omega_{0}} \omega_{0}=r+\frac{q}{l}+\frac{p}{m}
$$

Here $r \sim \hbar^{-1}$ is the main quantum number which controls the dimension of the quantum Hilbert space $\mathcal{P}_{r, q, p}$. The integers $q, p$ vary on the intervals (3.11), they control the fine structure of the scalar product (4.2) in $\mathcal{P}_{r, q, p}$.

In the case of "ground states", where $q=p=0$, the condition (4.16) becomes standard for the geometric quantization. In the "excited" case where $q \geq 1$ or $p \geq 1$, we observe something like an index contribution to the geometric quantization picture appearing due to an additional holonomy around the conical poles in $\Omega_{0}$. Because of these "excitations", the leaves $\Omega_{0}$ with quantized energies are distant from each other by $\frac{1}{l}$ or $\frac{1}{m}$ fractions of the parameter $\hbar$.

To conclude this section, let us discuss what quantum leaves of the algebra (2.4) are. To each element $\mathbf{F}$ of the algebra one can assign the corresponding operator $\mathbf{f}$ in the irreducible representation. This operator acts in the Hilbert space $\mathcal{P}_{r, q, p}$ of antiholomorphic sections over the phase space. Thus we can compose the function

$$
f \stackrel{\text { def }}{=} \frac{1}{K} \mathbf{f}(K)
$$

Here $K$ is the reproducing kernel (4.4) and the operator $\mathbf{f}$ acts by $\bar{z}$. The function $f$ (4.17) is called the Wick symbol of the operator $\mathbf{f}$, for more details see in 3, 34, 42, 43. The product of symbols in the sense of (4.6) corresponds to the product of operators. Moreover, one can reconstruct the operator by its symbol using the simple formula

$$
\mathbf{f}=f\left(\stackrel{2}{\mathbf{z}}^{*}, \stackrel{1}{\mathbf{z}}\right)
$$


where $\mathbf{z}^{*}$ is the operator of multiplication by $\bar{z}$ and $\mathbf{z}$ is the conjugate operator.

To generators of the algebra (2.4) we now can assign functions on the phase space:

$$
a_{j} \stackrel{\text { def }}{=} \frac{1}{K} \mathbf{a}_{j}(K) \quad(j=1,2), \quad a_{ \pm} \stackrel{\text { def }}{=} \frac{1}{K} \mathbf{a}_{ \pm}(K) .
$$

We can consider them as quantum analogs of the coordinate functions $A_{1}$, $A_{2}, A_{ \pm}=A_{3} \mp i A_{4}$ on classical symplectic leaves of the Poisson algebra (2.5).

Theorem 4.3. (a) The quantum coordinate functions obey the Casimir identities

$$
\begin{gathered}
k a_{1}+m a_{2}=E_{r, q, p}, \\
a_{+} * a_{-}=\left(a_{1}+\hbar\right) * \cdots *\left(a_{1}+m \hbar\right) * a_{2} *\left(a_{2}-\hbar\right) * \cdots *\left(a_{2}-l \hbar+\hbar\right) .
\end{gathered}
$$

Here $*$ is the quantum product (4.6).

(b) In the classical limit $\hbar \rightarrow 0$ (and $r \sim \hbar^{-1} \rightarrow \infty$ ) the quantum coordinate functions coincide with the classical coordinate functions (2.12) on the closure $\Omega(2.6)$ of the symplectic leaves $\Omega_{0}$.

Taking into account this theorem, we below identify the quantum phase space $\mathbb{S}^{2}$ with the closure $\Omega$ of the symplectic leaf (2.6), where $E=E_{r, q, p}$. We will call $\Omega$ endowed with this structure a quantum leaf.

Each element $\mathbf{F}$ of the algebra (2.4) can be represented as a polynomial in generators:

$$
\mathbf{F}=F(\mathbf{A}), \quad \mathbf{A}=\left(\stackrel{3}{\mathbf{A}_{+}}, \stackrel{2}{\mathbf{A}_{1}}, \stackrel{2}{\mathbf{A}_{2}}, \stackrel{1}{\mathbf{A}_{-}}\right)
$$

Here $F$ is a function on $\mathbb{R}^{4}$. The operation of multiplication of elements (4.19) determines a product operation $\odot$ in the algebra of polynomials over $\mathbb{R}^{4}$ :

$$
F(\mathbf{A}) G(\mathbf{A})=(F \odot G)(\mathbf{A})
$$

(see details in 32).

Following [32, 33], one can define the quantum restriction of the function $F$ onto the leaves $\Omega$ :

$$
\left.F\right|_{\hat{\Omega}} \stackrel{\text { def }}{=} \frac{1}{K} F(\mathbf{a})(K) .
$$

From [33] one known the following assertion. 
Theorem 4.4. (a) The quantum restriction (4.20) $\left.F \rightarrow F\right|_{\hat{\Omega}}$ is a homomorphism of algebras:

$$
\left.(F \odot G)\right|_{\hat{\Omega}}=\left.\left.F\right|_{\hat{\Omega}} * G\right|_{\hat{\Omega}} .
$$

The equivalent formula for the quantum restriction is

$$
\left.F\right|_{\hat{\Omega}}=F(a *) 1
$$

where a* are the operators of left multiplication by the quantum coordinate functions $a=\left(a_{+}, a_{1}, a_{2}, a_{-}\right)$(4.18) in the algebra (4.6).

(b) The asymptotics as $\hbar \rightarrow 0$ of the quantum restriction can be derived from

$$
\left.F\right|_{\hat{\Omega}}=F\left(a-i \hbar \operatorname{ad}_{-}(a)+O\left(\hbar^{2}\right)\right) 1=F(a)+\hbar e_{1}(F)+O\left(\hbar^{2}\right) .
$$

Here $\operatorname{ad}_{-}(\cdot)$ denotes the anti-holomorphic part of the Hamiltonian field: ad $_{-}(\cdot)=$ $i g^{-1} \partial(\cdot) \bar{\partial}$, where $g$ is the quantum metric (4.9). The $\hbar$-correction $e_{1}$ in (4.21) is the second order operator $e_{1}=\frac{1}{2}\left\langle R \frac{\partial}{\partial a}, \frac{\partial}{\partial a}\right\rangle$ determined by the symmetric tensor $R_{j l}=\operatorname{Re}\left(g^{-1} \partial a_{j} \bar{\partial} a_{l}\right)$.

\section{Coherent states and gyron spectrum}

In the Hilbert space $\mathcal{P}_{r, q, p}$ of anti-holomorphic sections of the Hermitian line bundle with the curvature $i \omega$ over the phase space $\Omega \approx \mathbb{S}^{2}$ we have the irreducible representation of the resonance algebra (2.4) by differential operators

$$
\begin{aligned}
& \mathbf{a}_{1}=\hbar(r m+p)-\hbar m \bar{z} \bar{\partial}, \quad \mathbf{a}_{2}=\hbar q+\hbar l \bar{z} \bar{\partial} \\
& \mathbf{a}_{+}=\hbar^{m} \prod_{j=1}^{m}(r m+p+j-m \bar{z} \bar{\partial}) \cdot \bar{z}, \quad \mathbf{a}_{-}=\frac{\hbar^{l}}{\bar{z}} \prod_{s=1}^{l}(q-s+1+l \bar{z} \bar{\partial}),
\end{aligned}
$$

where $\bar{\partial}=\partial / \partial \bar{z}$.

The unity section $1=\bar{z}^{0}$ is the vacuum vector for this representation in the sense that it is the eigenvector of the operators $\mathbf{a}_{1}, \mathbf{a}_{2}$ and it is annulled by the operator $\mathbf{a}_{-}$. Now let us take the vacuum vector $\mathfrak{P}_{0}$ in the original Hilbert space $\mathcal{L}=L^{2}\left(\mathbb{R}^{2}\right)$ which correspond to the representation (2.2):

$$
\mathbf{A}_{1} \mathfrak{P}_{0}=\hbar(r m+p) \cdot \mathfrak{P}_{0}, \quad \mathbf{A}_{2} \mathfrak{P}_{0}=\hbar q \cdot \mathfrak{P}_{0}, \quad \mathbf{A}_{-} \mathfrak{P}_{0}=0 .
$$


Definition 5.1. The coherent states of the algebra (2.4) is the holomorphic family of vectors $\mathfrak{P}_{z} \in \mathcal{L}$ defined by

$$
\mathfrak{P}_{z}=\sum_{n=0}^{r} \frac{q !}{(q+\ln ) !}\left(\frac{z}{\hbar^{l}}\right)^{n} \mathbf{A}_{+}^{n} \mathfrak{P}_{0}, \quad z \in \mathbf{C} .
$$

For each $a \in \Omega$ let us denote by $\boldsymbol{\Pi}_{a}$ the projection onto the one-dimensional subspace in $\mathcal{L}$ generated by $\mathfrak{P}_{z(a)}$. We call $\Pi_{a}$ a coherent projection.

Regarding these definitions, may be, it is useful to note the following: if one takes the Hilbert space $\mathcal{P}_{r, q, p}$ instead of $\mathcal{L}$ and the vacuum 1 instead of $\mathfrak{P}_{0}$, then instead of coherent states $\mathfrak{P}_{z}$ and the coherent projection $\boldsymbol{\Pi}_{a}$ one would see the reproducing kernel $K^{\#}(\cdot \mid z)$ and the probability function $p_{a}$.

In the following theorem we collect the basic properties of the coherent states $\mathfrak{P}_{z}$. In the general context of quantization theory, see more details in 32, 33, 34.

Theorem 5.1. (a) The scalar product of two coherent states coincides with the reproducing kernel (4.4):

$$
\left\|\mathfrak{P}_{z(a)}\right\|^{2}=K(a), \quad a \in \Omega
$$

(b) One has the resolution of unity by coherent projections:

$$
\frac{1}{2 \pi \hbar} \int_{\Omega} \boldsymbol{\Pi}_{a} d m(a)=\mathbf{I}_{r, q, p}
$$

Here $\mathbf{I}_{r, q, p}$ is the projection in $\mathcal{L}$ onto the Hilbert subspace $\mathcal{L}_{r, q, p}$ spanned by all vectors $\mathbf{A}_{+}^{n} \mathfrak{P}_{0}, n=0, \ldots, r$.

(c) The whole Hilbert space $\mathcal{L}$ is the direct sum of the irreducible subspaces:

$$
\mathcal{L}=\bigoplus_{\substack{r \geq 0 \\ 0 \leq q \leq l-1 \\ 0 \leq p \leq m-1}} \mathcal{L}_{r, q, p}
$$

(d) The coherent transform $\mathcal{L}_{r, q, p} \stackrel{\nu}{\rightarrow} \mathcal{P}_{r, q, p}$ defined by

$$
\nu(\psi)(\bar{z})=\left(\psi, \mathfrak{P}_{z}\right),
$$

has the inverse

$$
\nu^{-1}(\varphi)=\frac{1}{2 \pi \hbar} \int_{\Omega} \frac{\mathfrak{P} \otimes \varphi}{K} d m
$$


The mappings (5.2), (5.3) intertwine the representations (2.2) and (5.1) of the algebra (2.4).

(e) Let $\mathbf{F}$ be an element of the algebra (2.4) realized in the Hilbert space $\mathcal{L}$ via the generators (2.2) as in (4.19), and let $\mathbf{f}=\nu \circ \mathbf{F} \circ \nu^{-1}$ be the coherent transformation of $\mathbf{F}$ realized in the Hilbert space $\mathcal{P}_{r, q, p}$. Then the Wick symbol $f$ (4.17) coincides with the Wick symbol of $\mathbf{F}$ given by

$$
f(a)=\operatorname{tr}\left(\mathbf{F} \Pi_{a}\right), \quad a \in \Omega .
$$

The operators $\mathbf{F}, \mathbf{f}$ are reconstructed via their symbols using the formulas

$$
\mathbf{F}=F(\mathbf{A})=f\left(\stackrel{\mathbf{z}}{ }^{*}, \stackrel{1}{\mathbf{z}}\right), \quad \mathbf{f}=F(\mathbf{a})=f\left(\frac{2}{z}, \bar{z}^{*}\right),
$$

where $\mathbf{z}$ is the operator of complex structure (4.1), a are the operators of irreducible representation (5.1). The Wick symbol of the coherent projection $\boldsymbol{\Pi}_{a}$ is the probability function $p_{a}(4.10)$.

Now following [36, 44 - 48] we explain how to reduce the coherent transform to closed curves (Lagrangian submanifolds) in the phase space.

Let $\Lambda \subset \Omega$ be a smooth closed curve, which obeys the quantization condition

$$
\frac{1}{2 \pi \hbar} \int_{\Sigma}\left(\omega-\frac{\hbar}{2} \rho\right)-\frac{1}{2} \in \mathbb{Z}
$$

where $\omega=i g d \bar{z} \wedge d z$ is the quantum Kählerian form (4.9), $\rho=i \bar{\partial} \partial \ln g$ is the quantum Ricci form, and $\Sigma$ is a membrane in $\Omega$ with the boundary $\partial \Sigma=\Lambda$.

We choose certain parameterization of the curve expressed via the complex coordinate on the leaf as follows:

$$
\Lambda=\{z=z(t) \mid 0 \leq t \leq T\}
$$

and define the following basis of smooth functions on the curve:

$$
\phi^{(j)}(t)=\sqrt{\overline{\bar{z}(t)}} \exp \left\{-\frac{i}{\hbar} \int_{0}^{t}\left(\bar{\theta}-\frac{\hbar}{2} \bar{\varkappa}\right)\right\} \varphi^{(j)}(\bar{z}(t)), \quad j=0, \ldots, r .
$$

Here $\theta \stackrel{\text { def }}{=} i \hbar \partial \ln K$ and $\varkappa=i \partial \ln g$ are primitives of the quantum Kählerian form $\omega=d \theta$ and the quantum Ricci form $\rho=d \varkappa$, the integral in (5.6) is taken over a segment of the curve $\Lambda$, and the monomials $\varphi^{(j)}$ are defined in (3.15). 
Let us denote by $\mathcal{L}_{\Lambda}$ the vector subspace in $C^{\infty}(\Lambda)$ spanned by $\phi^{(j)}(j=$ $0, \ldots, r)$ and introduce the Hilbert structure in $\mathcal{L}_{\Lambda}$ by means of the following norm:

$$
\|\phi\|_{\Lambda} \stackrel{\text { def }}{=} \frac{1}{\sqrt[4]{2 \pi \hbar}}\left(\sum_{j=0}^{r}\left|\left(\phi, \phi^{(j)}\right)_{L^{2}}\right|^{2}\right)^{1 / 2},
$$

where the scalar product $(\cdot, \cdot)_{L^{2}}$ is taken in the $L^{2}$-space over $\Lambda$.

For any smooth function $\phi \in C^{\infty}(\Lambda)$ we define

$$
\mu_{\Lambda}(\phi)=\frac{1}{\sqrt[4]{2 \pi \hbar}} \int_{\Lambda} \phi(t) \sqrt{\dot{z}(t)} \exp \left\{\frac{i}{\hbar} \int_{0}^{t}\left(\theta-\frac{\hbar}{2} \varkappa\right)\right\} \mathfrak{P}_{z(t)} d t
$$

where $\mathfrak{P} \in \mathcal{L}$ are coherent states of algebra (2.4) corresponding to its $(r, q, p)$ irreducible representation.

Theorem 5.2. (a) The mapping $\mu_{\Lambda}$ defined by (5.8) is an isomorphism of Hilbert spaces

$$
\mu_{\Lambda}: \mathcal{L}_{\Lambda} \rightarrow \mathcal{L}_{r, q, p} \subset \mathcal{L}
$$

(b) Under the isomorphism (5.8) the representation of the algebra (2.4) in the Hilbert space $\mathcal{L}$ is transformed to the irreducible representation in the Hilbert space $\mathcal{L}_{\Lambda}$ :

$$
\mathbf{F} \rightarrow \mathbf{F}_{\Lambda} \stackrel{\text { def }}{=} \mu_{\Lambda}^{-1} \circ \mathbf{F} \circ \mu_{\Lambda} .
$$

(c) In the classical limit as $\hbar \rightarrow 0$ the Hilbert structure (5.7) coincides with the $L^{2}$-structure:

$$
\|\phi\|_{\Lambda}=\left(\int_{\Lambda}|\phi(t)|^{2} d t\right)^{1 / 2}+O(\hbar) .
$$

(d) Let $f$ be the Wick symbol (5.4) of the operator $\mathbf{F}$, then the asymptotics of the operator (5.9) as $\hbar \rightarrow 0$ is given by

$$
\mathbf{F}_{\Lambda}=\left.\mathcal{F}\right|_{\Lambda}-i \hbar\left(v+\frac{1}{2} \operatorname{div} v\right)+O\left(\hbar^{2}\right) .
$$

Here $\mathcal{F}=f-\frac{\hbar}{4} \Delta f$, by $\Delta$ we denote the Laplace operator with respect to the quantum Kählerian metric $g$, and $v=\left.\operatorname{ad}_{+}(\mathcal{F})\right|_{\Lambda}$ is the restriction to $\Lambda$ of the holomorphic part of the Hamiltonian field $\operatorname{ad}_{+}(\mathcal{F})=-i g^{-1} \bar{\partial} \mathcal{F} \cdot \partial$. 
The next terms of the asymptotic expansion (5.11) are also known (see in 36 ).

In Theorem 5.2, the curve $\Lambda$ is arbitrary except it has to obey the quantization condition (5.5).

Let us now choose $\Lambda$ specifically to be a closed curve on the energy level

$$
\Lambda \subset\{\mathcal{F}=\lambda\}
$$

and choose the coordinate $t$ to be time on the trajectory $\Lambda$ of the Hamiltonian field $\operatorname{ad}(\mathcal{F})$. Then $v=\left.\operatorname{ad}(\mathcal{F})\right|_{\Lambda}=\frac{d}{d t}, \operatorname{div} v=0$, and we have

$$
\mathbf{F}_{\Lambda}=\lambda-i \hbar \frac{d}{d t}+O\left(\hbar^{2}\right)
$$

This formula implies the asymptotics of eigenvalues of the operator $\mathbf{F}_{\Lambda}$ :

$$
\lambda+\hbar \frac{2 \pi k}{T}+O\left(\hbar^{2}\right)
$$

where $T=T(\lambda)$ is the period of the trajectory $\Lambda=\Lambda(\lambda)$ (5.12) and $\lambda$ is determined by the quantization condition (5.5).

Note that the contribution $\frac{2 \pi k}{T}$ added to $\lambda$ in (5.14) can be transformed to adding the number $k$ to the integer number on the right-hand side of condition (5.5). Thus one can omit the summand $\hbar \frac{2 \pi k}{T}$ in (5.16) without loss of generality.

Corollary 5.3. Let $\mathbf{F}$ be an operator commuting with the oscillator $\mathbf{E}(2.1)$. Up to $O\left(\hbar^{2}\right)$, the asymptotics of its eigenvalues $\lambda$ is determined by the quantization condition:

$$
\frac{1}{2 \pi \hbar} \int_{\Sigma}\left(\omega-\frac{\hbar}{2} \rho\right)-\frac{1}{2} \in \mathbb{Z}
$$

Here $\Sigma$ is a membrane in $\Omega$ with the boundary $\Lambda=\partial \Sigma(5.12)$; the curve $\Lambda$ is the energy level of the function $\mathcal{F}=f-\frac{\hbar}{4} \Delta f$, where $f$ is the Wick symbol of $\mathbf{F}$ and $\Delta$ is the Laplace operator. The operator $\Delta$ and the forms $\omega, \rho$ are generated by the quantum Kählerian metric $g$ (4.9).

Now we can apply the obtained results in studying quantum gyrons. Let one has the Hamiltonian of the type

$$
\mathbf{E}+\varepsilon \mathbf{B}
$$


where $\mathbf{E}$ is the oscillator (2.1) and $\mathbf{B}$ is a perturbation presented as a function in operators $\mathbf{b}, \mathbf{b}^{*}$,

$$
\mathbf{B}=\sum \beta_{\mu, \nu} \mathbf{b}^{* \nu} \mathbf{b}^{\mu}
$$

There is an operator averaging procedure [47, 48, which is a unitary transformation reducing $(5.16)$ (up to $O\left(\varepsilon^{N}\right)$ ) to the Hamiltonian

$$
\mathbf{E}+\varepsilon \mathbf{B} \sim \mathbf{E}+\varepsilon \mathbf{F}_{N}+O\left(\varepsilon^{N}\right), \quad\left[\mathbf{F}_{N}, \mathbf{E}\right]=0 .
$$

For instance, if $N=1$, then

$$
\mathbf{F}_{1}=\sum_{l \nu_{1}+m \nu_{2}=l \mu_{1}+m \mu_{2}} \beta_{\mu, \nu} \mathbf{b}^{* \nu} \mathbf{b}^{\mu}
$$

(see also the Appendix in [25]). For any $N \geq 1$ in (5.18), the operator $\mathbf{F}_{N}$, commuting with $\mathbf{E}$, is uniquely determined and can be presented in the form (4.19):

$$
\mathbf{F}_{N}=F_{N}(\mathbf{A})
$$

and after this in the form (5.4):

$$
\nu \circ \mathbf{F}_{N} \circ \nu^{-1}=F_{N}(\mathbf{a})=f_{N}\left(\frac{2}{z}, \frac{1}{z} *\right) .
$$

Thus the study of the operator (5.16) up to $O\left(\varepsilon^{N}\right)$ is reduced to the study of the properties of the operator (5.20) in each irreducible representation of the algebra (2.4).

The symbols $F_{N}$ or $f_{N}$ are gyron Hamiltonians. In the $(r, q, p)$-irreducible representation, the gyron is described by the operator $F_{N}(\mathbf{a})=F_{N}\left(\stackrel{3}{\mathbf{a}_{+}}, \underset{2}{\mathbf{a}_{1}}, \stackrel{2}{\mathbf{a}_{2}}, \stackrel{1}{\mathbf{a}_{-}}\right)$ acting in $\mathcal{P}_{r, q, p}$, where the generators a are given by (5.1).

In the semiclassical approximation $\hbar \rightarrow 0$ the gyron system can be reduced to (5.11) and even to (5.13) over the trajectory $\Lambda$ of the effective Hamiltonian $\mathcal{F}_{N}=f_{N}-\frac{\hbar}{4} \Delta f_{N}+O\left(\hbar^{2}\right)$ on the leaf $\Omega \approx \mathbb{S}^{2}$. The asymptotics of the gyron spectrum was described in Corollary 5.3 by means of the membrane versions (5.15) of the Bohr-Sommerfeld quantization condition.

The quantum Kählerian geometry (via the measure $d m$ and the forms $\omega, \rho)$ is essentially presented in all these results regarding the gyron spectrum.

The gyron is a model. It is very simple, since it arises from the "textbook" oscillator Hamiltonian. At the same time, it already contains many nontrivial aspects of the quantization theory and, of course, it has a variety of important 
physical applications. About more complicated models of this type and about further ideas on the quantum geometry we refer to [25, 32, 33], 51]-63.

Acknowledgements. The author is grateful to V. P. Maslov and E. M. Novikova for very useful discussions and help.

\section{References}

[1] I. E. Segal, Quantization of nonlinear systems, J. Math. Phys., 1 (1960), 468-488.

[2] G. W. Mackey, Mathematical Foundations of Quantum Mechanics, Benjamin, New York, 1963.

[3] J. R. Klauder, Continuous representation theory, J. Math. Phys., 4 (1963), 1055-1073.

[4] V. P. Maslov, Perturbation Theory and Asymptotic Methods, Moscow State Univ., 1965 (in Russian).

[5] J.-M. Souriau, Quantification geometrique, Comm. Math. Phys., 1 (1966), 374-398.

[6] B. Kostant, Quantization and unitary representations, Lect. Notes Math., 170 (1970), 87-208.

[7] A. Kirillov, Constructions of unitary irreducible representations of Lie groups, Vestnik Moskov. Univ. Ser. I Mat. Mekh., 2 (1970), 41-51 (in Russian); English transl. in Moscow Univ. Math. Bull.

[8] F. A. Berezin, Quantization, Izv. Akad. Nauk SSSR Ser. Mat., 38 (1974) 1116-1175; English transl., Math. USSR-Izv., 8 (1974), 1109-1165.

[9] F. Bayen, M. Flato, C. Fronsdal, A. Lichnerowicz, and D. Sternheimer, Quantum mechanics as a deformation of classical mechanics, Lett. Math. Phys., 1 (1975/77), 521-530.

[10] M. Rieffel, Deformation quantization for actions of $\mathbb{R}^{d}$, Mem. Amer. Math. Soc., 106 (1993), 1-93.

[11] H. Omori, Y. Maeda, and A. Yoshioka, Weyl manifolds and deformation quantization, Adv. Math., 85 (1991), 224-255. 
[12] B. Fedosov, A simple geometrical construction of deformation quantization, J. Diff. Geom., 40 (1994), 213-238.

[13] H. J. Groenewold, On the principles of elementary quantum mechanics, Physica, 12 (1946), 405-460.

[14] J. E. Moyal, Quantum mechanics as a statistical theory, Proc. Cambridge Phil. Soc., 45 (1949), 99-124.

[15] V. I. Arnold, V. V. Kozlov, and A. I. Neishtadt, Mathematical Aspects of Classical and Celestial Mechanics. In: Modern Problems in Math., Vol. 3, Moscow, VINITI, 1985, 5-303 (in Russian).

[16] V. M. Babich and V. S. Buldyrev, Asymptotic Methods in Problems of Diffraction of Short Waves, Nauka, Moscow, 1972 (in Russian).

[17] V. Guillemin, Symplectic spinors and partial differential equations, Colloques Intern. C.V.R.S., N237, Geom Sympl. \& Phys. Math., 1975.

[18] V. Guillemin and A. Weinstein, Eigenvalues associated with closed geodesics, Bull. Amer. Math. Soc., 82 (1976), 92-94.

[19] J. V. Ralston, On the construction of quasimodes associated with stable periodic orbits, Comm. Math. Phys., 51 (1976), 219-242.

[20] V. P. Maslov, Complex WKB-Method, Moscow, Nauka, 1976 (in Russian); English transl., Birkhäuser, Basel-Boston, 1994.

[21] Y. Colin de Verdiere, Quasi-modes sur les varietes Riemanniennes, Invent. Math., 43 (1977), 15-52.

[22] M. V. Karasev, Resonances and quantum method of characteristics, Intern. Conference "Differential Equations and Related Topics" (Moscow, 16-22 May, 2004), Petrovskii Seminar and Moscow Math. Society, Book of Abstracts, Publ. Moscow Univ., Moscow, 2004, 99-100 (in Russian).

[23] M. V. Karasev, Birkhoff resonances and quantum ray method, Proc. Intern. Seminar "Days of Diffraction - 2004", St. Petersburg University and Steklov Math. Institute, St. Petersburg, 2004, 114-126. 
[24] M. V. Karasev, Noncommutative algebras, nano-structures, and quantum dynamics generated by resonances, I. In: Quantum Algebras and Poisson Geometry in Mathematical Physics (M. Karasev, ed.), Amer. Math. Soc. Transl. Ser. 2, Vol. 216, Providence, RI, 2005, pp. 1-18. Preprint version in arXiv: math.QA/0412542

[25] M. V. Karasev, Noncommutative algebras, nano-structures, and quantum dynamics generated by resonances, II, Adv. Stud. Contemp. Math., 11 (2005), 33-56.

[26] M. V. Karasev, Formulas for noncommutative products of functions in terms of membranes and strings, Russ. J. Math. Phys., 2 (1994), 445462.

[27] M. V. Karasev, Geometric coherent states, membranes, and star products. In: Quantization, Coherent States, Complex Structures J.-P. Antoine et al., eds., Plenum, New York, 1995, 185-199.

[28] W. Fulton, Introduction to Toric Varieties, Ann. of Math. Stud., Princeton Univ., 131 (1993).

[29] G. W. Schwarz, Smooth functions invariant under the action of a compact Lie group, Topology, 14 (1975), 63-68.

[30] V. Poénaru, Singularités $C^{\infty}$ en présence de symmétrie, Lect. Notes Math., 510 (1976).

[31] A. S. Egilsson, Newton polyhedra and Poisson structures from certain linear Hamiltonian circle actions, Preprint version in arXiv: math.SG/0411398

[32] M. V. Karasev, Advances in quantization: quantum tensors, explicit star-products, and restriction to irreducible leaves, Diff. Geom. and Its Appl., 9 (1998), 89-134.

[33] M. V. Karasev, Quantum surfaces, special functions, and the tunneling effect, Lett. Math. Phys., 56 (2001), 229-269.

[34] M. Cahen, S. Gutt, and J. Rawnsley, Quantization of Kähler manifolds, I, J. Geom. Phys., 7 (1990), 45-62; II, Trans. Amer. Math. Soc., 337 (1993), 73-98; III, Lett. Math. Phys., 30 (1994), 291-305; IV, Lett. Math. Phys., 180 (1996), 99-108. 
[35] R. Brylinski and B. Kostant, Nilpotent orbits, normality, and Hamiltonian group actions, J. Amer. Math. Soc., 7 (1994), 269-298.

[36] M. V. Karasev, Quantization and coherent states over Lagrangian submanifolds, Russ. J. Math. Phys., 3 (1995), 393-400.

[37] M. V. Karasev and E. M. Novikova, Non-Lie permutation relations, coherent states, and quantum embedding. In: Coherent Transform, Quantization, and Poisson Geometry (M. Karasev, ed.), Amer. Math. Soc. Transl. Ser. 2, Vol. 187, Providence, RI, 1998, pp. 1-202.

[38] S. Bergmann, The kernel functions and conformal mapping, Math. Surveys Monographs, Vol. 5, Amer. Math. Soc., Providence, RI, 1950.

[39] V. Bargmann, On a Hilbert space of analytic functions and associated integral transform, Comm. Pure Appl. Math., 14 (1961), 187-214.

[40] M. V. Karasev, Integrals over membranes, transitions amplitudes and quantization, Russ. J. Math. Phys., 1 (1993), 523-526.

[41] S. Chern, Complex manifolds, Bull Amer. Math. Soc., 62 (1956), 101117.

[42] F. A. Berezin, Wick and anti-Wick symbols of operators, Mat. Sb., 86 (1971), 578-610 (in Russian); English transl. in Math. USSR-Sb., 15 (1971).

[43] F. A. Berezin, Covariant and contravariant symbols of operators, Izv. Akad. Nauk SSSR, Ser. Mat., 36 (1972), 1134-1167 (in Russian); English transl., Math. USSR Izv., 8 (1974), 1109-1165.

[44] M. V. Karasev, Connections over Lagrangian submanifolds and certain problems of semiclassical approximation, Zapiski Nauch. Sem. Leningrad. Otdel. Mat. Inst. (LOMI), 172 (1989), 41-54 (in Russian); English transl., J. Sov. Math., 59 (1992), 1053-1062.

[45] M. V. Karasev, Simple quantization formula. In: Symplectic Geometry and Mathematical Physics, Actes du colloque en l'honneur de J.M.Souriau (P. Donato et al., eds.), Birkhäuser, Basel-Boston, 1991, 234-243. 
[46] M. V. Karasev and M. B. Kozlov, Exact and semiclassical representation over Lagrangian submanifolds in $\mathrm{su}(2)^{*}$, $\mathrm{so}(4)^{*}$, and $\mathrm{su}(1,1)^{*}$, J. Math. Phys., 34 (1993), 4986-5006.

[47] M. V. Karasev and M. V. Kozlov, Representation of compact semisimple Lie algebras over Lagrangian submanifolds, Funktsional. Anal. i Prilozhen., 28 (1994), no. 4, 16-27 (in Russian); English transl., Functional Anal. Appl., 28 (1994), 238-246.

[48] M. V. Karasev, Quantization by means of two-dimensional surfaces (membranes): Geometrical formulas for wave-functions, Contemp. Math., 179 (1994), 83-113.

[49] A. Weinstein, Asymptotics of eigenvalue clusters for the Laplacian plus a potential, Duke Math. J., 44 (1977), 883-892.

[50] M. V. Karasev and V. P. Maslov, Asymptotic and geometric quantization, Uspekhi Mat. Nauk, 39 (1984), no. 6, 115-173 (in Russian); English transl. Russian Math. Surveys, 39 (1984), no. 6, 133-205.

[51] B. Mielnik, Geometry of quantum states, Comm. Math. Phys., 9 (1968), 55-80.

[52] A. Weinstein, Noncommutative geometry and geometric quantization. In: Symplectic Geometry and Mathematical Physics, Actes du colloque en l'honneur de J.-M.Souriau (P. Donato et al., eds.), Birkhäuser, BaselBoston, 1991, 446-462.

[53] A. Weinstein, Classical theta-functions and quantum tori, Publ. RIMS, Kyoto Univ., 30 (1994), 327-333.

[54] A. Connes, Noncommutative Geometry, Academic Press, London, 1994.

[55] M. V. Karasev and E. M. Novikova, Representation of exact and semiclassical eigenfunctions via coherent states. The Hydrogen atom in a magnetic field, Teoret. Mat. Fiz., 108 (1996), no. 3, 339-387 (in Russian); English transl. in Theoret. Math. Phys., 108 (1996).

[56] H. Omori, Y. Maeda, N. Miyazaki, and A. Yoshioka, Poincare-Cartan class and deformation quantization of Kähler manifolds, Comm. Math. Phys., 194 (1998), 207-230. 
[57] D. Sternheimer, Deformation quantization: Twenty years after. In: Particles, Fields, and Gravitation (J. Rembielinski, ed.), AIP Press, New York, 1998, 107-145.

[58] S. Gutt, Variations on deformation quantization. In: Conference Moshe Flato, 1999 (G. Dito and D. Sternheimer, eds.), Vol. 1, Kluwer Acad. Publ., 2000, 217-254.

[59] M. Kontsevich, Deformation quantization of algebraic varieties, Lett. Math. Phys., 56 (2001), no. 3, 271-294.

[60] Y. Manin, Theta functions, quantum tori, and Heisenberg groups, Lett. Math. Phys., 56 (2001), no. 3, 295-320.

[61] M. V. Karasev, Quantization and intrinsic dynamics. In: Asymptotic Methods for Wave and Quantum Problems (M. Karasev, ed.), Amer. Math. Soc. Transl. Ser. 2, Vol. 208, Providence, RI, 2003, pp. 1-32.

[62] M. V. Karasev, Intrinsic dynamics of manifolds: quantum paths, holonomy, and trajectory localization, Russ. J. Math. Phys., 11 (2004), 157176.

[63] M. V. Karasev and E. M. Novikova, Algebras with polynomial commutation relations for a quantum particle in electric and magnetic fields. In: Quantum Algebras and Poisson Geometry in Mathematical Physics (M. Karasev, ed.), Amer. Math. Soc. Transl. Ser. 2, Vol. 216, Providence, RI, 2005, pp. 19-135. 